VOL. $14(1976), 299-302$.

\title{
On a problem of Kurt Mahler concernin! binomial coefficents
}

\section{lan S. Williams}

Recently Kurt Mahler asked: for which natural numbers $N$ is the least common multiple of all the binomial coefficients $\left(\begin{array}{l}N \\ k\end{array}\right)$ the product of the primes less than or equal to $N$ ? We obtain a formula for the least common multiple of all the binomial coefficients of any natural number $N$ and hence show that 2,11 , and 23 are the only solutions to Mahler's problem.

Write $\mathrm{LCM}_{N}$ for the least common multiple of the binomial coefficients $\left(\begin{array}{l}N \\ k\end{array}\right)$ of $N, k=0, \ldots, N$.

LEMMA. Let $N$ be any natural number and $p_{i}^{r_{i}}$ be a prime power such that $p_{i}^{r_{i}} \leq N+1<p_{i}^{r_{i}^{+1}}$. Then

$$
\operatorname{LCM}_{N}=\left(\prod_{i} p_{i}^{r}\right)^{i} /(N+1)
$$

where the product is over all primes $p_{i} \leq N+1$.

Proof. Let $q$ be any prime power less than or equal to $N+1$.

Received 9 January 1976. Communicated by Jennifer R.S. Wallis. The author wishes to thank Professor Kurt Mahler and Dr Jennifer Seberry Wallis for their help and encouragement in preparing this note. Calculations which suggested the final formulas were programmed using the Research School of Physical Sciences, Australian National University, Dec-lo system. 
Then $q$ divides one and only one of

$$
N+1, N, N-1, \ldots, N+2-q .
$$

Hence $q$ divides the numerator and not the denominator of

$$
\frac{(N+1) N(N-1) \ldots(N+2-q)}{1.2 \cdot 3 \ldots(q-1)}=\left(\begin{array}{c}
N \\
q-1
\end{array}\right)(N+1) \text {. }
$$

So if $q=p_{i}^{{ }^{i}}$, where $p_{i}^{r_{i}} \leq N+1<p_{i}^{r_{i}^{+1}}$, then $p_{i}^{{ }^{r}} \mid(N+1)\left(\begin{array}{c}N \\ q-1\end{array}\right)$. But this is true for any prime power $p_{i}{ }_{i}$. Hence

$$
\prod_{i} p_{i}^{i} \mid(N+1) \mathrm{LCM}_{N}
$$

It remains to show that if $p \mid q=p^{\alpha}$, then no higher power of $p$ than $p^{a}$ divides the left-hand side of (1). This could only happen if some power of $p$, say $p^{b}<p^{a}$ occurred more times in the numerator than in the denominator; this clearly cannot happen, as $p^{b}$ divides exactly $p^{a-b}-1$ other terms in both the numerator and denominator.

Hence we have the result.

THEOREM. Let $N$ be a natural number. Suppose the least common multiple of the binomial coefficients of $N$ is the product of the primes less than or equal to $N$. Then $N$ is 2, 11 , or 23.

Proof. Write ${ }^{\mathrm{LCM}}{ }_{N}=\prod_{i} p_{i}$ for the least common multiple of the primes less than or equal to $N$. Now using the previous lemma,

$$
\operatorname{LCM}_{N}=\left(\prod_{j} p_{j}^{r}\right) /(N+1)
$$

where $p_{j}$ runs over all primes less than or equal to $N+1$, and where $p_{j}^{r} \leq N+1<p_{j}^{r_{j}^{+I}}$. Hence, unless $N+1$ is a prime or prime power,

$$
N=\prod_{j} p_{j}^{r_{j}^{-1}}-1
$$


Clearly $r_{j}=1$ for any prime greater than $\sqrt{N+1}$, and so such a prime does not appear in (2). Similarly for any prime less than or equal to $\sqrt{N+1}$ the condition $p_{j}^{r} \leq N+1<p_{j}^{r^{+1}}$ ensures that $r_{j} \geq 2$, and so it does appear in (2). Thus every prime less than $\sqrt{N+1}$ is a factor of $N+1$ and not of $N$. Hence $N$ is a prime.

Therefore we consider the primes $N$ of the form

$$
N=\prod_{p_{j}}^{r} j^{-1}-1
$$

in which every prime $p_{j} \leq \sqrt{N+1}$ occurs and for which $p_{j}^{r} \leq N+1<p_{j}^{r} j^{+1}$

First we show $N+I$ cannot be divisible by 3 to a power greater than or equal to 2 . Suppose the contrary; then

$$
N=2^{a} 3^{2+b} k-1, a>0, b \geq 0,
$$

satisfies all the assumptions. Thus $3^{3+b} \leq N+1<3^{4+b}$ and $a \geq 3$ (since $\left.2^{a+1} \leq N+1<2^{a+2}\right)$. But this means

$$
\begin{gathered}
3^{3+b} \leq 2^{a} 3^{2+b} k<3^{4+b}, \\
3 \leq 2^{a} k<3^{2}
\end{gathered}
$$

which can only be satisfied for $a=3, k=1$. In this case $N=2^{3} 3^{2+b}-1,2^{a+1}=16 \leq N+1<2^{a+2}=32$ and so $b<0$, which is a contradiction.

Hence the power of 3 is 0 or 1 . Now consider $b=0$; then

$$
N=2^{a}-1
$$

and $3>\sqrt{N+1}$; so $N=7$ or 3 which clearly do not satisfy the assumption that $2^{a+1} \leq N+1<2^{a+2}$.

It remains to consider natural numbers $N=2^{a} 3-1$. We must have

$$
3^{2} \leq N+1<3^{3}, 2^{a+1} \leq N+1<2^{a+2} \text {, and } 5>\sqrt{N+1} \text {. }
$$


Clearly $N=11=2^{2} \cdot 3-1$ and $N=23=2^{3} \cdot 3-1$ are the only natural numbers which satisfy all the assumptions, when $N+1$ is not a prime or prime power.

If $N+1$ is a prime power, $p_{i}^{r_{i}}$, then from the lemma, $p_{i}$ will not divide $\mathrm{LCM}_{N}$ and so $\mathrm{LCM}_{N}$ is not a product of the primes less than or equal to $N$.

If $N+1$ is prime, then $N$ can only equal 2 , as $N$ must be prime using the same argument as above. Clearly $\mathrm{LCM}_{2}=\frac{2 \cdot 3}{2}=2$ satisfies the assumptions.

Hence we have the result.

Director's Unit,

Research School of Physical Sciences, Australian National University, Canberra, ACT. 